\title{
Die Heiligtümer der Nation. Abraham Lincoln, Erinnerungs- kultur und die „nationale Einheit“ der USA
}

\author{
Benedikt Kapferer \\ Kerngebiet: Zeitgeschichte \\ eingereicht bei: Univ.-Prof. Mag. Dr. Dirk Rupnow \\ eingereicht im: SoSe 2018 \\ Rubrik: SE-Arbeit
}

\begin{abstract}
The Nation's Hallows. Abraham Lincoln, Culture of Remembrance and the „National Unity" of the USA

For US-American nation building, Abraham Lincoln played such a vital role that he became a major focal point in US politics, society and culture. His significance even reached dimensions of religious worship, which represents a key characteristic of US culture of remembrance. Especially in modern, pluralistic democracies, such an intertwining of nationhood and religion may cause societal tensions. This paper deals with Lincoln's efforts to create a religion of the nation, establishing a hegemonic culture of remembrance. Based on the concept of "civil religion", it investigates the meaning and implication of religious elements, particularly the "Pledge of Allegiance", and the role of history in the contemporary USA.
\end{abstract}


"As is always the case in any society trying to master the most conflicted elements of its past, healing and justice had to happen in history and through politics."

\section{Einleitung}

In der Geschichte der Vereinigten Staaten von Amerika spielten Kontinuitäten und Traditionen im kollektiven Bewusstsein und in der öffentlichen Darstellung stets eine wichtige Rolle. Seit der Unabhängigkeitserklärung im Jahr 1776 war die Rhetorik in der politischen Kultur des Landes geprägt von einer Verehrung von gemeinsamen Werten und Prinzipien wie etwa Freiheit und Gerechtigkeit. Im 19. Jahrhundert zerfielen die USA allerdings kurzzeitig mit der Sezession und der Gründung der Konföderierten Staaten von Amerika, was schließlich zum Bürgerkrieg zwischen 1861 und 1865 führte. Dieser wird gemeinhin als erster modern geführter Massenkrieg bezeichnet ${ }^{2}$ und ist mit über 620.000 Toten der verlustreichste militärische Konflikt in der Geschichte der USA. ${ }^{3}$ Gerade in der zweiten Hälfte des 19. Jahrhunderts war er ein „tiefer Epocheneinschnitt“4 und ein Wendepunkt für die USA in einem Zeitalter des aufkommenden Nationalismus und des nation building. Dieser Epocheneinschnitt war ein Ereignis, das der zukünftigen Gesellschaft auf vielfache Weise in Erinnerung blieb. So bildet er für die Wahrnehmung einer "nationalen Einheit" einen omnipräsenten Gegenstand der Erinnerungskultur. Der Topos der nationalen Einheit, der gesamte historische Kontext des US-amerikanischen Bürgerkrieges sowie die sich darauf beziehende Erinnerungskultur sind untrennbar mit der Person Abraham Lincolns ${ }^{5}$ verbunden. Nagler schreibt dem 16. Präsidenten der USA mit folgenden Worten einen besonderen Stellenwert zu: „In seiner zum Mythos gewordenen Person bündeln sich die wesentlichen Merkmale der amerikanischen

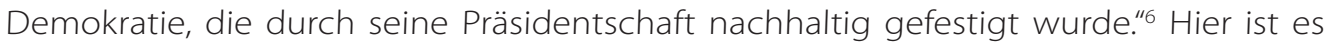
nicht nur bemerkenswert, dass seine Person zum "Mythos" wurde, sondern auch, dass sich darin die "wesentlichen Merkmale der amerikanischen Demokratie“ widerspiegeln und - noch entscheidender - er diese nachhaltig verankerte. Ähnlich werden dem Bürgerkrieg in den USA meist die Topoi „unity and continuity“ auferlegt.?

Angesichts der Omnipräsenz des Bürgerkrieges und der Person Lincolns in der Erinnerungskultur der USA nehmen diese historischen Sachverhalte eine besondere Stellung in der Öffentlichkeit ein. Sie prägen damit bis in die Gegenwart maßgeblich die Public History sowie den Prozess des kollektiven Erinnerns der USA. Insofern erscheint Abraham Lincoln in seinem Wirken und seiner Bedeutung für die nationale Einheit der USA mit einer besonderen Nachhaltigkeit verknüpft zu sein. Die vorliegende Arbeit unter-

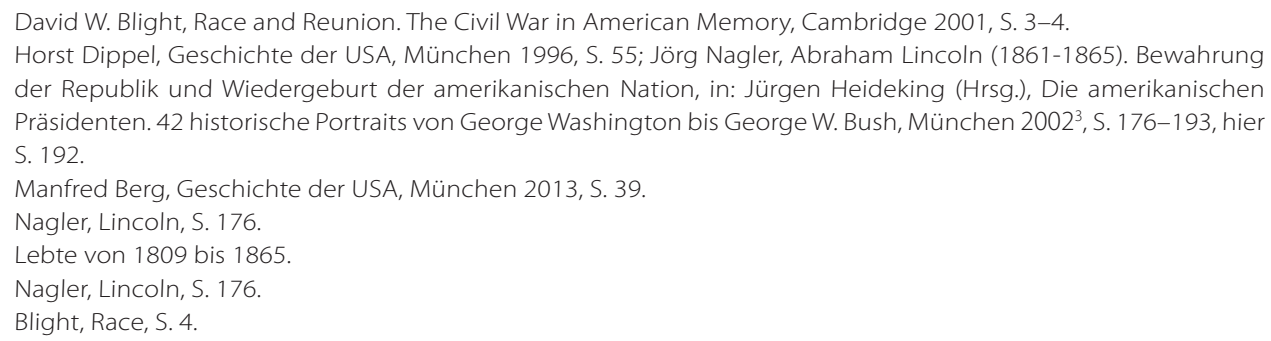


sucht diese Nachhaltigkeit und die lang andauernde Wirkung der Person Lincolns in Bezug auf den Topos der nationalen Einheit der USA. Es wird den Fragen nachgegangen, welche Rolle das Erinnern für Abraham Lincoln, die Einheit und Identität der USA in seiner Zeit spielte und welche Maßnahmen er dafür in seiner Politik umsetzte. Darüber hinaus beschäftigt sich die Arbeit mit der Frage, wie sich die (vermeintliche) nationale Einheit - basierend auf Lincoln - in der politischen Kultur und der Öffentlichkeit der USA bis in die Gegenwart widerspiegelt. Dabei wird von der These ausgegangen, dass Lincoln sich der Bedeutung und Wirkung des kollektiven Erinnerns bewusst war und deshalb versuchte, diese für den Erhalt der Union bzw. die Konstruktion der Nation zu nutzen und zu fördern. Lincolns Bemühungen sind somit eng mit der öffentlichen Darstellung von Geschichte und der Erinnerungskultur der USA verbunden, was sich im von Robert Bellah geprägten Konzept der Civil Religion zeigt. In dieser Kultur der Public History nimmt der sogenannte "Pledge of Allegiance”, das gesetzlich festgeschriebene Treuegelöbnis, eine Sonderposition ein. Als performativer Akt der Erinnerung steht er indirekt mit Lincoln in Verbindung, propagiert ein Gefühl der nationalen Einheit und regt gleichzeitig eine kritische Betrachtung des Themas von außen an. Insofern lässt sich ausgehend von der Person Lincolns und den historischen Ereignissen des Bürgerkrieges in einer Untersuchung der kulturellen Praxis der Erinnerung ein zeitlicher Bogen bis in die Gegenwart spannen und die Kontinuitäten und Traditionslinien in der Geschichte der USA hinterfragen.

Die Erfahrung des Bürgerkrieges war zweifelsfrei maßgeblich verantwortlich für die politische und soziale Entwicklung der Gesellschaft sowie die Herausbildung einer hegemonialen nationalen Identität der USA. In der vorliegenden Untersuchung steht allerdings nicht das Ereignis selbst im Fokus, sondern vielmehr die politische Sprache und Rhetorik von Abraham Lincoln. Der hier gewählte Zugang konzentriert sich in einem ersten Schritt darauf, wie Lincoln selbst über Erinnerung und nationale Einheit dachte, wie er sie für sein politisches Ziel - den Erhalt der Union - zu nutzen wusste und schließlich auch, wie er mit diesem Diskurs die nachfolgende Erinnerungskultur prägte. Dafür wird v. a. seine seltener untersuchte und bereits sehr früh gehaltene Rede vor dem Young Men's Lyceum aus dem Jahr 1838 berücksichtigt. Im Zentrum der Analyse stehen dabei linguistische Aspekte sowie das kollektive Gedächtnis. Dadurch kommen besonders seine Ziele, aber auch seine Vorstellungen über die Mechanismen einer nationalen Einigung zum Ausdruck. Ausgehend davon geht es in einem zweiten Schritt darum, welche Implikationen ein solcher Diskurs über nationale Einheit und Erinnerungskultur in einer demokratischen Gesellschaft besitzt. Es geht dabei um die Herausforderungen und Differenzen, die bei einer Beschwörung der nationalen Einheit auftreten können, und letztlich auch um die Frage, wie mit diesen Differenzen oder einer Kritik umgegangen wird. Im Fokus liegen somit eine kulturelle Praxis der nationalen Einheit, soll heißen eine Performativität der Nation, was unter dem Schlagwort doing nation betrachtet werden kann, und die Frage, was dies über eine pluralistische, demokratische Gesellschaft im 21. Jahrhundert aussagt. Für einen solchen Zugang braucht es verschiedene Konzepte, die für die Analyse die notwendigen Untersuchungsinstrumente liefern. Dabei ist der Zusammenhang zwischen Geschichte und Öffentlichkeit 
zentral. Als Grundlage dient das Konzept der bereits erwähnten Public History. Diese beschäftigt sich „mit öffentlichen (Re-)Präsentationen von Geschichte [...], analysiert diese und dekonstruiert darin zum Ausdruck kommende Geschichtsbilder, um den öffentlichen Gebrauch und Missbrauch der Historie zu untersuchen". Der "Öffentliche Gebrauch" von Geschichte manifestiert sich wesentlich in der Geschichts- bzw. Erinnerungskultur einer Gesellschaft. Konkret ist bei den USA das Konzept der Civil Religion ein Teil der nationalen Erinnerungskultur und somit der Public History zuzuschreiben. Dieser Zusammenhang ist wesentlich, um die Bedeutung Abraham Lincolns und die Frage der nationalen Einheit in den USA zu verstehen. Darüber hinaus ist Benedict Andersons Konzept der Nation als imagined community fundamental. Nach Benedict Anderson ist die Nation lediglich „imagined", also vorgestellt, weil sich deren Mitglieder untereinander nie alle kennen würden, aber "in the minds of each lives the image of their communion“. Dieses "image“, das gemeinsame mentale Bild und Gefühl der Nation, stiftet somit eine Gemeinschaft und die dazugehörige Vorstellung einer inneren sozialen Kohäsion. Ein solches Bild und Gefühl gilt es im Bestreben einer nationalen Einigungsbewegung zu vermitteln und über längere Zeit zu erhalten. Hier wird die Idee der Nachhaltigkeit bei Lincoln, seinem Werk und seiner Wirkung greifbar und besonders relevant. Dabei ist allerdings von einem weiten Begriffsverständnis von solchen „Bildern”, wie sie Anderson anspricht, auszugehen. Die Bilder der Nation werden in der Sphäre der Public History hergestellt und reproduziert. Diese werden somit in der Geschichts- und Erinnerungskultur gepflegt und in Erinnerungsorten bewahrt und gezeigt. In Bezug auf die Frage der nationalen Einheit bedeutet dies schließlich, die Bilder der Nation zu beleuchten und deren Zustandekommen und Bedeutung zu entschlüsseln, wobei u. a. Jan und Aleida Assmann wesentliche Beiträge zur Theorie leisteten. ${ }^{10}$ Anderson verdeutlicht die Bindung der Menschen zur Nation in einem imaginativen Prozess anhand der Sprache, die genutzt wird. So würden Begriffe aus dem Vokabular der Familie, wie etwa "motherland, fatherland, homeland“, das Gefühl einer naturgemäBen Verbindung vermitteln. ${ }^{11}$ Dies betont die zentrale Rolle, die Sprache in der Analyse einer Rede einnimmt. Allgemein ist der Bürgerkrieg gegen Ende der Sattelzeit im Kontext der nationalen Einigungsbewegungen, wie sie z. B. in Italien und Deutschland stattfanden, zu sehen. ${ }^{12}$ Wie Angelika Schaser grundlegend die Nation und Nationalgeschichte seit dem Ende des 18. Jahrhunderts als „politisches Projekt“13 bezeichnet, kann die Herstellung von nationaler Einheit als das politische Projekt Lincolns schlechthin angesehen werden. Basierend auf diesen Konzepten versucht die vorliegende Arbeit eine Dekonstruktion der mentalen Strukturen und kulturellen Praktiken der nationalen Einheit durchzuführen.

8 Martin Lücke/Irmgard Zündorf, Einführung in die Public History, Göttingen 2018, S. 24.

9 Benedict Anderson, Imagined Communities. Reflections on the Origin and Spread of Nationalism, London-New York 2006, S. 6.

10 Astrid Erll, Kollektives Gedächtnis und Erinnerungskulturen. Eine Einführung, Stuttgart-Weimar 20112, S. 27-29.

11 Jenny Barrett, Shooting the Civil War. Cinema, History and American National Identity, London 2009, S. 14.

12 Nagler, Lincoln, S. 193.

13 Angelika Schaser, Nation, Identität und Geschlecht. Nationalgeschichtsschreibung und historische Frauen- und Geschlechterforschung, in: Karin Hagemann/Jean Quataert (Hrsg.), Geschichte und Geschlechter. Revisionen der neueren deutschen Geschichte, Frankfurt am Main 2008, S. 64-91, hier S. 66. 
In Anbetracht des tiefen Epocheneinschnittes und des Mythos, den der Bürgerkrieg bzw. Abraham Lincoln darstellen, ist es kaum verwunderlich, dass es über diese Epoche der US-Geschichte eine unermesslich große Menge an Forschungsliteratur und historischen sowie geschichtskulturellen Darstellungen gibt. Allgemein lässt sich über den Stand der Forschung sagen, dass die Themen des Bürgerkrieges, die Person und die Präsidentschaft Abraham Lincolns, das nationale Selbstbild der USA und die Erinnerungskultur weitgehend bearbeitet wurden. Für die vorliegende Arbeit bilden die Aufsätze von Jörg Nagler und Kurt Müller einen zentralen Bestandteil. ${ }^{14}$ Nach wie vor grundlegend sind die Monografien von Barry Schwartz ${ }^{15}$ und David W. Blight, da sie detailliert den Zusammenhang zwischen Lincolns Person bzw. dem Bürgerkrieg und einer kollektiven nationalen Erinnerung herausarbeiten. Zum Konzept der Civil Religion lieferte Robert Bellah in einem Aufsatz des Jahres $1967^{16}$ die Grundlage, auf welche sich nach wie vor andere Darstellungen beziehen. ${ }^{17}$ Hervorzuheben ist, dass sämtliche einschlägige Diskussionen des Konzeptes der Civil Religion Abraham Lincoln eine besondere Rolle darin zuschreiben, ihn aber nie mit dem „Pledge of Allegiance" als ein Beispiel dieser Civil Religion in Verbindung bringen. ${ }^{18}$ Generell kommt der "Pledge " wenig in den Diskussionen vor. Lediglich Publikationen zum „Pledge” selbst ordnen ihn in den Kanon der Civil Religion ein, verbinden ihn aber nicht mit Lincoln oder seiner Rede vor dem Young Men's Lyceum. Zugegebenermaßen liegt zwischen dieser Rede Lincolns des Jahres 1838 bzw. Lincolns Leben und der erstmaligen Veröffentlichung des „Pledge of Allegiance" im Jahr 1892 eine gewisse zeitliche Distanz. Nichtsdestotrotz ist diese Verknüpfung für die Frage der nationalen Einheit und der Rolle Lincolns in Bezug auf die Erinnerungskultur besonders relevant. In dieser zeitlichen Distanz liegen nämlich gerade auch die angesprochene Nachhaltigkeit und Kontinuität, die von Lincoln ausgingen und sich vom Kontext des Bürgerkrieges bis in die Gegenwart in der Öffentlichkeit und Kultur der USA verfestigt haben. In dieser Arbeit sollen diese Kontinuitäten als Mechanismen der Erinnerungskultur und als Manifestationen der nationalen Einheit untersucht und die Verknüpfungen aufgezeigt werden.

Um diese Kontinuitäten und Entwicklungslinien darzustellen, wählt die Arbeit einen chronologischen Aufbau. So stehen zu Beginn der Kontext des Bürgerkrieges und Abraham Lincoln selbst, wobei Lincoln und sein „politisches Projekt” zur Sprache kommen. Hier stehen die Analyse und Diskussion der Rhetorik Lincolns und die Rolle der Erinnerung für die Konstruktion der Nation im Fokus. Danach konzentriert sich die Darstellung auf den Zusammenhang der Civil Religion und der nationalen Einheit in den USA sowie auf die Bedeutung des „Pledge of Allegiance“. Ein abschließender Ausblick thematisiert den Umgang mit Kritik an der Nation in einer demokratischen Gesellschaft.

14 Kurt Müller, Abraham Lincoln. The Emergence, Appropriation, and Contestation of an American Icon, in: Günther Leypoldt/Bernd Engler (Hrsg.), American Cultural Icons. The Production of Representative Lives, Würzburg 2010, S. 383-409.

15 Barry Schwartz, Abraham Lincoln and the Forge of National Memory, Chicago-London 2000

16 Robert Bellah, Civil Religion in America, in: Daedalus 96 (1967), Nr. 1, S. 1-21.

17 Annika Hvithamar/MargitWarburg, Introducing Civil Religion, Nationalism and Globalisation, in: Annika Hvithamar/ Margit Warburg/Brian Arly Jacobsen (Hrsg.), Holy Nations and Global Identities. Civil Religion, Nationalism, and Globalisation, Leiden-Boston 2009, S. 1-17.

18 Nagler, Lincoln, S. 192; Müller, Emergence, S. 393; Bellah, Religion, S. 10. 


\section{Lincoln, Erinnerung und das Projekt der Nation}

Abraham Lincoln wurde im Jahr 1809 in Kentucky geboren. Er ging lediglich für ein Jahr in die Schule und galt als Autodidakt. Im Jahr 1832 meldete er sich als Freiwilliger im Black-Hawk-Krieg und 1834 zog er nach einer zwei Jahre zuvor gescheiterten Wahl erfolgreich in das Repräsentantenhaus von Illinois ein. 1836 wurde er nach einem Selbststudium der Rechtswissenschaften zur Anwaltskammer zugelassen. ${ }^{19}$ In seiner Tätigkeit als Rechtsanwalt konnte er sich einen Ruf erarbeiten, der ihn in der Öffentlichkeit als "Honest Abe" mit Eigenschaften wie Fleiß, Arbeitsethos, Bescheidenheit und Volksnähe darstellen ließ.20 Dabei konnte er mit seiner Herkunft aus armen Pionierverhältnissen und seinem sozialen Aufstieg die Ideale eines self-made man und somit auch den American Dream verkörpern. ${ }^{21}$ Lincoln eignet sich deshalb als nationale Identifikationsfigur und Personifikation US-amerikanischer Werte. In der Frage der Sklaverei zählte Lincoln zwar nicht zu den Abolitionist*innen, lehnte die Sklaverei aber ab²2 und wirkte "extrem polarisierend".23 Er hatte eine "eher gemäßigte Position" und trat öffentlich weniger für ihre Abschaffung, sondern viel mehr für das Verhindern ihrer Ausdehnung ein. ${ }^{24}$ Dadurch sei es ihm kurz nach Beginn des Bürgerkrieges gelungen, die sogenannten Border States, also Bundesstaaten an der Grenze zwischen Norden und Süden, auf die Seite des Nordens zu bringen. ${ }^{25}$ Nach Auflösung von Lincolns Whig Party wurde er als Kandidat der 1854 neu gegründeten Republikanischen Partei26 im Jahr 1860 zum Präsidenten der USA gewählt. Mit der Sezession und dem Bürgerkrieg konfrontiert war sein oberstes Kriegsziel der Erhalt der Union und weniger die Abschaffung der Sklaverei.. ${ }^{27}$ Kurz gesagt war Lincoln ein geschickter politischer Stratege, der wusste, welche Ziele er zu welcher Zeit verfolgen musste, um die Mehrheit der Wahlberechtigten, die politischen Repräsentanten sowie einzelne Bundesstaaten hinter sich zu bringen.

In der Frage danach, wie Lincoln den Erhalt der Union auf längere Sicht zu gewährleisten und ein Gefühl der nationalen Einheit zu vermitteln versuchte, ist es notwendig, die Rolle der Erinnerung zu untersuchen. Laut Berg sei ein Grund für den Ausbruch des Bürgerkrieges die Haltung vieler Südstaatler*innen gewesen, ihre Loyalität zum jeweiligen Bundesstaat über die Treue zur Union zu stellen, wie z. B. der General der US-Armee Robert E. Lee aus Virginia in die Konföderation folgte. ${ }^{28}$ Insofern ging es Lincoln darum, diese Loyalität und Treue auf das politische Gebilde der Union auszuweiten. Wie Nagler schreibt, war "Lincolns ganzes Denken und Handeln darauf ausgerichtet, das Erbe der Founding Fathers - die in Unabhängigkeitserklärung und

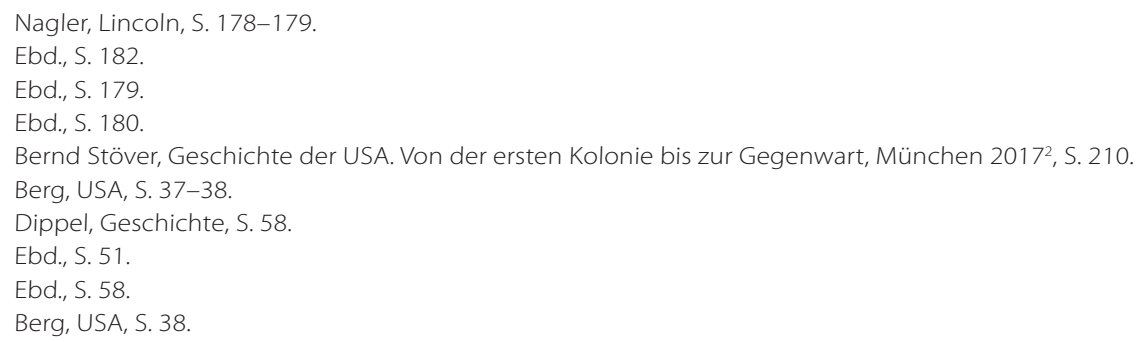


Verfassung niedergelegten Werte und Prinzipien der Republik - zu retten und erneut voll zur Geltung zu bringen“.29 In diesem Zitat stecken mehrere besonders wichtige Aspekte, nämlich die Kombination von Denken und Handeln, das Erbe der Gründerväter sowie die auf die Unabhängigkeitserklärung und Verfassung zurückgehenden Werte und Prinzipien der Republik. Diese Elemente nahm Lincoln als wesentliche Bezugspunkte für sein politisches Projekt der Konstruktion einer Nation. In der Bedeutung und Wirkung der Erinnerung sah er das Mittel, dies zu erreichen.

Es besteht kein Zweifel, dass Erinnerung ein fundamentaler Akt ist. Für Individuen, Gruppen und Gesellschaften ist sie eine bedeutende Kulturtechnik, welche es erlaubt, sich auf Elemente der Vergangenheit zurück zu besinnen und dadurch Identitäten, Traditionslinien, Sinn- und Deutungskonstruktionen herzustellen. Alexis de Tocqueville stellte in seinem in zwei Teilen 1835 und 1840 veröffentlichten Klassiker „Democracy in America" besonders interessante Überlegungen über die Rolle der Erinnerung in einer demokratischen Gesellschaft im Vergleich zu einer aristokratischen Gesellschaft an. Er identifiziert den Individualismus als ein wesentliches Merkmal einer Demokratie und sieht ihn in gewisser Weise als Gefahr für den Verlust sozialer Kohäsion an. ${ }^{30}$ Aufgrund einer fehlenden sozialen Mobilität schreibt er aristokratischen Nationen zu, dass Menschen - er spricht lediglich von Männern - darin eine größere Verbundenheit zu ihren Vorfahr*innen und Ursprüngen hätten. Im originalen Wortlaut heißt es: „He willingly assumes duties toward his ancestors and descendants, frequently sacrificing his personal pleasures for the sake of those beings who have gone before and who have yet to come." ${ }^{\prime 11}$ Besonders wichtig ist die Verbundenheit zwischen den Generationen und den zeitlichen Dimensionen, die er entwirft. Er spricht von einer stärkeren affektiven Vernetzung zwischen den Vorfahr*innen und den Nachkommen. Deshalb schreibt er Mitgliedern der aristokratischen Gesellschaftsform die Bereitschaft zu, sich für die Vorund Nachfahr*innen zu opfern und persönliche Vergnügungen hintanzustellen. Darüber hinaus merkt er über gesellschaftliche Instititutionen Folgendes an: „In addition, aristocratic institutions achieve the effect of binding each man closely to several of his fellow citizens."32 Er spricht somit von einer stärkeren sozialen Kohäsion, die von Institutionen in aristokratischen Systemen ausgehen. In seinen Überlegungen behandelt er die grundlegende soziologische Frage, was eine Gesellschaft zusammenhält. Dies ist wiederum die Kernfrage für Abraham Lincoln und den gesamten Kontext der USA im 19. Jahrhundert, die sich schließlich im Bürgerkrieg gewaltsam ausdrückte. Demokratische Gesellschaften charakterisiert er hingegen gänzlich anders:

„Among democratic nations, new families constantly emerge from oblivion, while others fall away; all remaining families shift with time. The thread of time is ever ruptured and the track of generations is blotted out. Those who have gone

29 Nagler, Lincoln, S. 176

30 Alexis de Tocqueville, Democracy in America and Two Essays on America, London 2003, S. 587-588.

31 Ebd., S. 588.

32 Grace Y. Kao/Jerome E. Copulsky, The Pledge of Allegiance and the Meanings and Limits of Civil Religion, in: Journal of the American Academy of Religion 75 (2007), Nr. 1, S. 121-149, hier S. 141. 
before are easily forgotten and those who follow are still completely unknown. Only those nearest to us are of any concern to us." ${ }^{133}$

Durch den oben bereits angesprochenen Individualismus und dadurch, dass immer wieder neue Familien aus der Vergessenheit oder Nichtbeachtung („oblivion”) emporkommen, gibt es laut Tocqueville in demokratischen Nationen weniger Bewusstsein und Interesse für die eigene Vergangenheit, Geschichte oder Vor- und Nachfahr*innen. Besonders drastisch klingen dabei die Formulierungen vom "Zerreißen des Fadens der Zeit" und „Auslöschen der Spur der Generationen“, die für ihn Folgen eines gesellschaftlichen Vergessens aufgrund einer fehlenden Erinnerung sind. Bemerkenswert ist auch, wie er mit der sprachlichen Verwendung von Bildern wie Familie, Generationen oder Vor- und Nachfahr*innen eine zeitliche Kontinuität herstellt. An dieser Stelle sei erneut an die von Anderson angestellten Überlegungen zur sprachlichen Konstruktion einer Nation mithilfe von Begriffen wie "Familie", "Vaterland" oder "Mutterland" verwiesen. ${ }^{34}$ Darüber hinaus ist speziell die Trias von Vorfahr*innen, der aktuellen Generation und den Nachfahr*innen - gekoppelt an die zeitlichen Dimensionen der Vergangenheit, Gegenwart und Zukunft - ein wiederkehrendes und bedeutsames Motiv.

Des Weiteren spricht Tocqueville aufgrund der sozialen Mobilität („as each class closes up to the others and merges with them ${ }^{\prime 35}$ ) von einer gesellschaftlichen Durchmischung, welche die Mitglieder einer Gesellschaft sich untereinander gleichgültig werden und sie voneinander entfremden lässt. Den Gegensatz zwischen den beiden Gesellschaftsformen fasst er prägnant zusammen: „Aristocracy had created a long chain of citizens from the peasant to the king; democracy breaks down this chain and separates all the links." ${ }^{136}$ Folglich schließt er: "Thus, not only does democracy make men forget their ancestors but also hides their descendants and keeps them apart from their fellows." ${ }^{137}$ Daraus resultiere schließlich eine Isolierung und Abschottung der Menschen untereinander $($,[ $[\ldots]$ brings them back to themselves and threatens in the end to imprison them in the isolation of their own hearts"). ${ }^{38}$ Kurz gesagt gibt es laut Tocqueville in einer Demokratie im Vergleich zur Aristokratie keinen wirklichen Zusammenhalt oder eine class identity. Die Menschen vergessen ihre Vorfahr*innen und kümmern sich nicht um ihre Nachkommen. In diesem individualistischeren Bild der Gesellschaft gibt es keine Verbindungen zwischen den Menschen, keine Erinnerung, sondern nur ein Vergessen. Wichtig ist dabei, dass er eine enge Verbindung zwischen sozialer Kohäsion und Erinnerung bzw. der Rolle der Vergangenheit für eine Gesellschaft ausarbeitet. Genau diese von Tocqueville - beinahe mit einem trauernden und wehmütigen Ton unterlegten Punkte, die er an der demokratischen Nation beschreibt, gilt es für Lincoln herzustellen.

Abraham Lincoln war der von Tocqueville geschilderte Zusammenhang zwischen Geschichte, Erinnerung und Gesellschaft bewusst und er versuchte diesen für sein politi-

33 Kao/Copulsky, Pledge, S. 589.

34 Barrett, Civil War, S. 14.

35 Tocqueville, Democracy, S. 589.

36 Ebd.

37 Ebd.

38 Ebd. 
sches Projekt - die Herstellung eines Gefühls der nationalen Einheit - zu nutzen. Dies zeigt sich bei einer Analyse seiner öffentlichen Reden, wobei die bereits erwähnte Rede aus dem Jahr 1838 im Zentrum steht. Lincolns Ansprache vor dem Young Men's Lyceum in Springfield ist zwar keine große Unbekannte, allerdings steht sie meist im Schatten der "Gettysburg Address", der "House-Divided-Speech" oder den „Inaugurations". In Anbetracht ihres zeitlichen Kontexts von 1838 zählt sie wohl zu einem der frühesten politischen Auftritte Lincolns, der sich erst 1834 mit dem Einzug ins Repräsentantenhaus von Illinois eine Stimme auf der offiziellen politischen Bühne verschaffte. Der Titel der Rede, "The Perpetuation of Our Political Institutions", ist ein vielsagendes Element. Der Begriff der "Fortdauer" (perpetuation) enthält so bereits die zeitliche Komponente und etabliert damit das Konzept der Nachhaltigkeit. Der Hintergrund ist die in den Jahren zuvor sich weiter radikalisierende und gewaltbereiter werdende Gesellschaft, konkret der 1837 verübte Lynchmord am abolitionistischen Pfarrer und Journalisten Elijah Lovejoy. ${ }^{39}$ Lincoln kritisiert in der Rede den Zustand der aktuellen politischen Kultur und die Stimmung in der Gesellschaft. Er identifiziert einen „increasing disregard for law which pervades the country“. Dies äußere sich im Ignorieren von den legitimierten rechtlichen Institutionen, den Gerichten und Gesetzen durch das Auftreten von „savage mobs" ${ }^{40}$ Allgemein sei für das "American People" eine Entfremdung und Distanzierung von der Regierung die natürliche Folge dieses "mobocratic spirit” in der Gesellschaft. Diese Stimmung stelle eine Gefahr für das Überleben der staatlichen Ordnung und der politischen Institutionen dar. ${ }^{41}$ Dies spricht auch der Titel der Rede an, da Lincoln über das Weiterbestehen der politischen Institutionen reflektiert bzw. dieses als besonders gefährdet darstellt. Nach Lincoln-Biograph David Herbert Donald sprach er damit v. a. eine "Hyperemotionalität in der Politik" an. ${ }^{42}$ Angesichts seines jungen Alters von nicht einmal 29 Jahren ist es bemerkenswert, welche Weitsicht Lincoln hatte und wie nachhaltig und vorausschauend er dachte. Zusätzlich wirkt er als besonders staatstragend, da er über die soziale Kohäsion und das Fortbestehen der Union sprach. Dies ist die Stimmungslage, die Lincoln dazu bewegte, diese Rede zu halten und seine darin enthaltenen Ansichten und Vorschläge gegen diese Entwicklungen vorzustellen.

Seine Ideen zum Umgang mit diesen Problemen basieren im Wesentlichen auf der Bedeutung der Erinnerung und der Pflege oder Kultur der Erinnerung. Lincoln beginnt die Rede mit einem Lob an die ursprüngliche Konzeption der USA und an die Besonderheit der noch nie zuvor dagewesenen Staats- und Regierungsform, die für zivile und religiöse Freiheit sorge. Er spricht dabei mehrmals von einem wir, welches er als "legal inheritors of these fundamental blessings" identifiziert.43 Damit stellt er bereits die Verbindung zu den Gründervätern der USA her und führt die Idee eines Erbes ein. Als Nächstes schildert er nostalgisch die „Aufgabe“ ("task"), welchen „a once hardy, brave, and patriotic, but now lamented and departed race of ancestors" ausübte. Er sah

39 Nagler, Lincoln, S. 180

40 Abraham Lincoln, Address to the Young Men's Lyceum of Springfield, Illinois, January 27, 1838, in: David S. Reynolds (Hrsg.), Lincoln's Selected Writings, New York-London 2015, S. 8-14, hier S. 9.

41 Ebd., S. 11.

42 David Herbert Donald, Lincoln, New York 1995, S. 80.

43 Lincoln, Lyceum, S. 8. 
Folgendes als deren Aufgabe: „[...] to uprear upon its hills and valleys, a political edifice of liberty, and equal rights."44 Der Begriff edifice hat zwei Bedeutungen: Einerseits ist es wortwörtlich ein "großes, imposantes Gebäude“, andererseits ein „komplexes System von Vorstellungen“.45 Lincoln verlagert in seiner Verwendung des Begriffes political edifice die Idee eines Gebäudes auf die von den Gründervätern etablierten Glaubensvorstellungen und Prinzipien. Damit baut er in seiner Sprache ein Gebäude und somit ein bildliches Konstrukt der Nation. Ein Gebäude ist ein abgetrennter und klar definierter Raum, der ein Gefühl von Einheit und Geschlossenheit vermittelt. Dieses Bild griff Lincoln im Jahr 1858 bei seiner Nominierung zum Präsidentschaftskandidaten in seiner berühmten „House Divided Speech" in folgendem Wortlaut wieder auf: „A house divided against itself cannot stand." ${ }^{\prime 6}$ Hier setzt er das gesamte Land, die Union und das politische System in einer Bibelreferenz (Matthäus 12,25)47 mit einem Haus gleich und bekräftigt, dass dieses im geteilten Zustand nicht bestehen könne. Wie Barrett anmerkt, vermittelt er mit dieser Metapher für die Nation „a sense of home“48 Der Wortlaut des political edifice in der Rede des Jahres 1838 erfüllt zweifelsfrei eine ähnliche Funktion.

Lincoln führt in „The Perpetuation of Our Political Institutions” weiter aus, dass das Erbe der Gründerväter eine Verpflichtung beinhalte: "This task of gratitude to our fathers, justice to ourselves, duty to posterity, and love for our species in general, all imperatively require us faithfully to perform." ${ }^{49}$ Es gehe darum, Dankbarkeit oder Erkenntlichkeit den Vätern, Gerechtigkeit der eigenen Generation und Arbeit, Pflicht oder einen Dienst der Nachwelt sowie allgemein Liebe für die Spezies entgegenzubringen. Wie auch Tocqueville zuvor bedient sich Lincoln der Trias von Vergangenheit (fathers), Gegenwart (ourselves) und Zukunft (posterity) und stellt dadurch Kontinuitäten und Traditionslinien her. Es scheint fast, als hätte Lincoln Tocquevilles Ansichten über einen schwächeren Zusammenhalt in einer Demokratie gelesen und sich davon inspirieren lassen. Zwar konnte Lincoln diese Ausführungen aus dem zweiten, erst 1840 veröffentlichten Teil aus Tocquevilles magnum opus zur Zeit der Rede im Jahr 1838 noch nicht gekannt haben. Dennoch zeigt dies, welche Rolle die Erinnerung für Lincoln hatte und wie bewusst er sich ihrer möglicher Wirkung war. Es war damit sein Ziel, wortwörtlich eine Tradition aufzubauen, nämlich ein nachhaltiges Weitergeben und Pflegen von Werten über das eigene Leben hinaus. Er propagiert ein Bewusstsein über die zeitlichen Dimensionen, das nicht nur auf die eigene Existenz, sondern eben auch auf die vor- und nachfolgenden Generationen bezogen sein sollte. Diese Bemühungen lassen sich mit geschichtsdidaktischen Überlegungen von Hans-Jürgen Pandel und Jörn Rüsen verbinden. Bei Ersterem ist die Unterscheidung zwischen "gestern - heute - morgen" als „Temporalbewusstsein" eine wesentliche Form des Geschichtsbewusstseins eines Indi-

\footnotetext{
44 Lincoln, Lyceum, S. 9.

45 Edifice, in: English Oxford Dictionary Online, O. D., [https://en.oxforddictionaries.com/definition/edifice], eingesehen 28. 9. 2018.

46 Abraham Lincoln, House Divided Speech at Springfield, Illinois, 1858, in: Reynolds (Hrsg.), Selected Writings, S. 129-135, hier S 129.

47 Nagler, Lincoln, S. 181.

48 Barrett, Civil War, S. 15

49 Lincoln, Lyceum, S. 9.
} 
viduums ${ }^{50}$ Letzterer schreibt historischem Erzählen eine Bedeutung als kommunikativer Akt einer "Sinnbildung über Zeiterfahrung" zu. ${ }^{51}$ Im kommunikativen Akt der Rede konstruiert Lincoln selbst eine historische Erzählung der Nation und schafft ein Angebot für eine Sinnbildung und Identifikation mit dieser. Wie Rüsen festhält, „eröffnet das historische Erzählen dem durch Zeiterfahrung irritierten Handeln des Menschen eine durch Zeiterfahrung gesicherte Zukunftsperspektive“.52 Dieses „irritierte Handeln des Menschen" kann in Lincolns Kontext in einer allgemeinen Irritation und Desillusionierung im nationalen, gesellschaftlichen Klima gesehen werden. Das historische Erzählen bietet schließlich die dazugehörige Zukunftsperspektive. Interessanterweise spricht Rüsen auch davon, dass das historische Erzählen die drei zeitlichen Dimensionen „synthetisiert" und ein „übergreifender Sinn“ als eine „Richtungsbestimmung, einer zeitspezifischen Orientierungsgröße (intentionaler) Bestandteil der menschlichen Lebenspraxis wird".53 Eine solche Synthese der zeitlichen Dimensionen und eine Sinnbildung erfolgt auch bei Lincoln. Sie basieren auf der Erinnerung und der Besinnung auf die Vergangenheit als gemeinsame Geschichte und die Nachwelt als gemeinsame Zukunft im Rahmen eines gemeinsamen Bewusstseins der Nation.

Rüsens angesprochener „übergreifender Sinn“, der zu einem „Bestandteil der menschlichen Lebenspraxis wird", spielt in Lincolns Überlegungen eine wesentliche Rolle. Er fordert eine solche Lebenspraxis ein, denn ihm zufolge verpflichte dieses emotional aufgeladene temporale Bewusstsein zu einem treuen Handeln („,all imperatively require us faithfully to perform"). ${ }^{54}$ Diese Ergebenheit und Treue solle sich somit in Taten äußern. Dabei ist die Wortwahl to perform signifikant, als sie einen Konnex zur Handlungstheorie bzw. dem Konzept der Performativität zulässt. Der mentale Prozess des Erinnerns manifestiert sich schließlich als Performanz in der Gedenk- und Erinnerungskultur eines Individuums oder einer Gruppe. Folglich können performative Akte der Erinnerungskultur in ihrer Bedeutung eine Performativität des Nationsgedankens darstellen. In diesem Sinne fordert Lincoln eine Bekräftigung der Loyalität zu den Werten und Institutionen des Staates und damit eine Hingabe zur Nation. Es geht um eine Forderung und Förderung einer Praxis, die als doing nation verstanden werden könnte und um die Herstellung nationaler Einheit bemüht ist. Wie diese Bekräftigung der Loyalität und Treue zur Nation sowie die angesprochene Performanz aussehen sollten, arbeitet er in der Rede weiter aus. Als konkrete Maßnahme schlägt er eine Art Treueschwur oder -gelöbnis vor. Im originalen Wortlaut entwickelt er diese Idee folgendermaßen:

"Let every American, every lover of liberty, every well wisher to his posterity, swear by the blood of the Revolution, never to violate in the least particular, the laws of the country; and never to tolerate their violation by others." ${ }^{\prime 5}$

50 Hans-Jürgen Pandel, Geschichtsunterricht nach PISA. Kompetenzen, Bildungsstandards und Kerncurricula, Schwalbach/Ts. 2005, S. 8 bzw. S. 10-11.

51 Jörn Rüsen, Historisches Lernen. Grundlagen und Paradigmen, Köln-Weimar-Wien 1994, S. 79.

52 Ebd., S. 80 .

53 Ebd

54 Lincoln, Lyceum, S. 9.

55 Ebd., S. 11; Hervorhebung des Verfassers. 
Lincoln geht es darum, die Beziehung jedes Individuums zu den Gesetzen des Landes zu stärken, indem sie auf das „Blut der Revolution“ schwören, niemals gegen die Gesetze zu verstoßen und andere nicht zu tolerieren, die dies tun. Ferner fordert er:

"[...] let every American pledge his life, his property, and his sacred honor;-let every man remember that to violate the law is to trample on the blood of his father, and to tear the character of his own and his children's liberty."56

Mit diesen Worten bringt er den Schwur oder das Gelöbnis (pledge) auf eine noch höhere, unmittelbarere und individuellere Ebene. Er spricht das Individuum direkt an, da es sich beim eigenen Leben, dem Besitz und der "heiligen Ehre“ verpflichten solle. Es solle jedem Mann bewusst sein, dass ein Verstoß gegen die Gesetze ein „Trampeln“ auf dem „Blut seines Vaters", ein „Riss im eigenen Charakter" und „in der Freiheit der Kinder" bedeute. Damit stellt Lincoln einen universellen Anspruch her. Universell ist dieser durch das erneut vorkommende Motiv der Trias der zeitlichen Dimensionen mit den familialen Verbindungen. Er stiftet eine Kontinuität, welche sich wieder auf die familiäre Ebene bezieht und die Vorfahr*innen, das Individuum selbst und die Nachfahr*innen involviert. Dies koppelt die Herausbildung einer Identität der eigenen Person und Familie an eine kollektive, nationale Identität und an die Gesetze und Werte des Staates. An dieser Stelle gelangt Lincoln zum Herzstück seiner Rede:

"Let reverence for the laws, be breathed by every American mother, to the lisping babe, that prattles on her lap-let it be taught in schools, in seminaries, and in colleges;-let it be written in Primmers, spelling books, and in Almanacs;-let it be preached from the pulpit, proclaimed in legislative halls, and enforced in courts of justice. And, in short, let it become the political religion of the nation; and let the old and the young, the rich and the poor, the grave and the gay, of all sexes and tongues, and colours and conditions, sacrifice unceasingly upon its altars. ${ }^{157}$

Lincoln weitet darin die geforderte Verehrung der Gesetze auf sämtliche gesellschaftliche Bereiche und Gruppen aus. In Verbindung mit dem Treueschwur soll die von ihm propagierte Haltung die verschiedensten Instanzen der Sozialisation, gesellschaftliche und politische Institutionen, Strukturen und Kulturgüter durchdringen. Die Gesetzestreue soll von der amerikanischen Mutter dem noch lispelnden Kleinkind beigebracht werden, sie soll gelehrt werden in Schulen, Seminaren und Colleges, niedergeschrieben in Lesebüchern / Fibeln und in Almanachen, gepredigt von der Kanzel, verkündet von gesetzgebenden Institutionen und durchgesetzt werden von Gerichten. Diese Haltung solle zu einer Art „politischen Religion“ der Nation werden. Diese richte sich an sämtliche soziale Schichten, inklusive "the rich and the poor", "all sexes and tongues" sowie "colours and conditions". Wie heterogen Lincoln diese Inklusionsidee jedoch tatsächlich fasste, wird expressis verbis nicht weiter geschildert. Nichtsdestotrotz ist zu konstatieren, dass Lincolns Ausführungen unter Berücksichtigung der Intersektionalität von Class, Race und Gender grundsätzlich einen progressiven Eindruck wecken. Von allen sozialen

56 Lincoln, Lyceum, S. 11; Hervorhebung des Verfassers.

57 Ebd., S. 11-12. 
Schichten und Gruppen fordert er somit ein "Opfer" auf dem „Altar" dieser politischen Religion ein. Speziell Lincolns Sprache macht eine enge Verstrickung des Politischen mit dem Religiösen evident: Er vereinnahmt typisch religiöse Elemente und Räume der Kirche, wie etwa die Kanzel (pulpit) als Ort der Predigt oder den Altar als Ort für das religiöse Ritual der Opfergabe. Damit stellt Lincoln eine Verbindung zwischen ursprünglich religiösen Räumen und Bedeutungsfeldern auf der einen und der staatlichen, zivilen und säkularen Ebene der Gesetze und Verfassung auf der anderen Seite her. Nagler verweist auf Lincolns Idee der "politischen Religion" ${ }^{\text {"58 }}$ und stellt die darin enthaltene Vermischung von religiöser und politischer Dimension in einen Zusammenhang mit dem Konzept der Civil Religion. ${ }^{59}$ Das folgende Kapitel behandelt eben dieses und bringt Lincolns Forderung nach einem Treueschwur mit dem „Pledge of Allegiance“ in Verbindung.

Zusammenfassend lässt sich die Erinnerung als zentraler Bestandteil in Abraham Lincolns Projekt der Nation beschreiben. Lincoln war sich ihrer Bedeutung und Wirkung bewusst und versuchte, diese für die Herstellung eines Gefühls der nationalen Einheit zu nutzen. Ausgehend von der Verfassung, den Gesetzen und den Werten der USA stellte er Kontinuitäten zwischen den Gründervätern, seiner eigenen Generation - welche mit den inneren Differenzen und der Spaltung des Staates konfrontiert war - sowie der Nachwelt her. Das Beispiel der Rede vor dem Young Men's Lyceum verdeutlicht diese fundamentale Rolle der Erinnerung für Lincoln. Selbstverständlich ist sie nur ein kleiner Ausschnitt aus Lincolns Zugang zur Erinnerung, zumal er sie bereits sehr früh in seiner politischen Karriere hielt. Hier sei lediglich auf die vielen anderen bedeutenden und vielfach behandelten Beispiele, die ebenso die Rolle der Erinnerung für die nationale Einheit beleuchten, verwiesen. Dazu gehören die "Gettysburg Address"60 oder die berühmte Formulierung der in seiner ersten Inaugurationsrede $1861 \mathrm{im}$ Zuge der Sezession der Südstaaten beschworenen "mystic chords of memory“,, in welcher die Erinnerung als "mystische Klänge" ein einheitsstiftendes Element besitzt und die Union zusammenhalten soll. Insofern ist die Rede vor dem Young Men's Lyceum ein vielsagendes Beispiel für Lincolns Rhetorik, die er mit ähnlichen Motiven und Bildern später weiter verfolgte. Er war bemüht, eine gesamtgesellschaftliche Praxis zu etablieren, in der die Menschen nicht nur der Vorfahr*innen gedenken, sondern sich in einem Schwur auf den Staat und die nationalen Werte emotional binden. Wortwörtlich sollten sie im performativen Akt eines Schwurs ihre Treue und Loyalität zur Nation und den damit einhergehenden Gesetzen und Werten herstellen. Wenngleich Lincoln den pledge nicht weiter konkretisierte, stellen seine Ausführungen eine bemerkenswerte Maßnahme zur Verankerung einer nationalen Einheit im Staat dar. Diese Konkretisierung kam schließlich mit dem „Pledge of Allegiance“, welcher im Rahmen des Konzeptes der Civil Religion weiteren Aufschluss über die Mechanismen des Nationalismus gibt.

58 Nagler, Lincoln, S. 180

59 Ebd., S. 192.

60 Berg, USA, S. 41; Nagler, Lincoln, S. 192; Barrett, Civil War, S. 15

61 Abraham Lincoln, First Inaugural Address, March 4, 1861, in: Reynolds (Hrsg.), Selected Writings, S. 228-234, hier S. 234; Nagler, Lincoln, S. 192. 


\section{Civil Religion und der "Pledge of Allegiance“}

\subsection{Das Konzept der Civil Religion im Zeichen der Erinnerungskultur}

Im Jahr 1967 lieferte Robert Bellah grundlegende Überlegungen über die Bedeutung von religiösen Elementen in der politischen Kultur der USA. ${ }^{62}$ Seiner Beobachtung nach gebe es eine religiöse Dimension in der Öffentlichkeit, welche sich in verschiedenen Einstellungen, Symbolen und Ritualen ausdrücke. Er bezeichnete diese als Civil Religion und beschrieb damit die Omnipräsenz religiöser Elemente im zivilen und politischen Leben. ${ }^{63}$ Zum Beispiel habe die Civil Religion bis zum Bürgerkrieg das biblische Motiv des Exodus mit Moses auf die US-amerikanische Revolution und George Washington projiziert. ${ }^{64}$ Mit dem Bürgerkrieg seien neue Motive, wie etwa "death, sacrifice, and rebirth", aufgekommen und v. a. von Abraham Lincoln - nicht zuletzt durch seine "Gettysburg Address" und seine dramatische Ermordung - symbolisiert worden. ${ }^{65}$ Dies spiegelt Blights Einschätzung wider, dass es nach wie vor schwierig sei, den Bürgerkrieg ohne eine Sentimentalität zu betrachten. ${ }^{66}$ Bellah ordnet die "Gettysburg Address" einem „Lincolnian ,New Testament" zu und beschreibt damit die Kategorie der "civil scriptures" - eine Art literarisch-religiöser Kanon mit den zentralen Texten der Nation. ${ }^{67}$ So enthalte die Civil Religion der USA einen Komplex verschiedener Kategorien, wie etwa "its own prophets and martyrs, its own sacred events and sacred places, its own solemn rituals and symbols". ${ }^{\prime \prime}$ In den folgenden Jahren etablierte sich das Konzept für die Analyse des Zusammenhangs von Religion, Nation(alismus), Identität und sozialer Kohäsion. Im Allgemeinen definieren Hvithamar und Warburg den Begriff als „phenomena for which the nation is the focal point of sacralisation". ${ }^{9}$ Reeh beschreibt ihn treffend als

"narration of selected historical experiences of the US that become woven into the fabric of the present (internally as well as externally) as an officially recognised myth of the American state".70

Schließlich habe die Civil Religion die Funktion, die Gesellschaft zusammenzuhalten.11 Die Nation dient demnach als Bezugspunkt für eine Heiligung und spielt mit der Erzählung historischer Erfahrungen eine besondere Rolle für eine nationale Einheit und eine Fortdauer nationaler Mythen in der Gegenwart. Basierend auf Bellah konkretisiert Müller das Konzept:

62 Bellah, Religion, S. 3.

63 Ebd., S. 4.

64 Ebd., S. 9.

65 Ebd., S. 10.

66 Blight, Race, S. 4.

67 Bellah, Religion, S. 10.

68 Ebd., S. 18.

69 Hvithamar/Warburg, Introducing, S. 1.

70 Niels Reeh, American Civil Religion as State-Mythology, in: Annika Hvithamar/Margit Warburg/Brian Arly Jacobsen (Hrsg.), Holy Nations and Global Identities. Civil Religion, Nationalism, and Globalisation, Leiden-Boston 2009, S. 79-97, hier S. 96.

71 Ebd., S. 85. 
"[It] has its, holy scriptures', its, ritual calendar' in national commemoration days, its sanctified sites of memory in national cemeteries and monuments, as well as its heroes, martyrs and saints. ${ }^{172}$

Gewissermaßen gibt es somit eine Reihe von Heiligtümern der Nation. Mit dem Verweis auf "national commemoration days" und "sanctified sites of memory" spricht Müller wesentliche Elemente einer Erinnerungskultur an. So kann festgehalten werden, dass die US-amerikanische Civil Religion als religiöse Aufladung der Nation eine Form der nationalen Erinnerungskultur vorzugeben scheint.

Die Vermischung von religiösen und staatlich-nationalen Elementen zeigt sich an verschiedenen Beispielen. So gehörten die Unabhängigkeitserklärung oder Lincolns "Gettysburg Address" zu den sacred texts und analog dazu der Nationalfriedhof von Gettysburg zu den sacred places. ${ }^{73}$ Diese zentralen Erinnerungsorte bezeichnet Hebel kritisch als "commercially marketed shrine of pilgrimage and collective worship which anticipated twentieth-century memory tourism and business".74 Ein ähnliches Beispiel für einen shrine of pilgrimage ist das Mount Rushmore National Memorial in South Dakota. Im 1941 fertiggestellten „nationalen Monument" steht Abraham Lincoln in einer Reihe mit den Präsidenten Washington, Jefferson und Theodore Roosevelt und symbolisiert die „Einheit der amerikanischen Nation".75 Der gemeinhin als shrine of democracy ${ }^{76}$ bekannte Ort sieht seine Aufgabe darin, „to communicate the founding, expansion, preservation, and unification" der USA anhand der kolossalen Statuen. ${ }^{77}$ Der Ausdruck shrine of democracy kann dabei als Inbegriff der Civil Religion, der religiösen Aufladung der politischen Kultur und nationaler Mythen, verstanden werden. Bei genauerer Betrachtung der Geschichte des Nationalparks Mount Rushmore steht allerdings die Tradition der Diskriminierung und des Ausschlusses der autochthonen Völker Amerikas in starkem Kontrast zur hegemonial weißen Geschichtserzählung der USA. Diese berücksichtige nämlich kaum den Protest der Sioux-Stämme gegen den Bau des Monuments und die damit einhergehende Verletzung ihrer Rechte. ${ }^{78}$ Dazu hält Boime fest:

"Consistent with the pattern of destruction characteristic of national exploration and empire building, the creation and the dedication of the Mount Rushmore monument was one more symbol of the white man's racist and intolerant Indian policy." ${ }^{\prime 79}$

72 Müller, Emergence, S. 385.

73 Gerald Parsons, Perspectives on Civil Religion, Burlington-Sydney 2002, S. 1.

74 Udo J. Hebel, Sites of Memory in U.S.-American Histories and Cultures, in: Astrid Erll/Ansgar Nünning (Hrsg.), Cultural Memory Studies. An International and Interdisciplinary Handbook, Berlin-New York 2008, S. 47-60, hier S. 54.

75 Alexander Emmerich, Geschichte der USA, Stuttgart 2008, S. 166.

76 National Park Service, 6. 7. 2011, [https://www.nps.gov/moru/learn/news/thousands-celebrate-at-the-shrine-ofdemocracy.htm], eingesehen 17. 10. 2018.

77 National Park Service, 19. 4. 2017, [https://www.nps.gov/moru/learn/historyculture/index.htm], eingesehen 17. 10. 2018.

78 Albert Boime, Patriarchy Fixed in Stone. Gutzon Borglum's "Mount Rushmore", in: American Art 5 (1991), Nr. 1, S. 142-167, hier S. 156. 
Dieser Umstand deutet die Brisanz einer (religiösen) Beschwörung der Nation in einer demokratischen Gesellschaft an.

Ob die Civil Religion der heutigen USA den Vorstellungen Lincolns entsprechen würde, bleibt aufgrund mangelnder Konkretisierungen seinerseits dahingestellt. Nichtsdestotrotz ist der Zusammenhang von Lincolns Forderung nach einer political religion, in welcher primär die Werte und Gesetze des Staates verehrt werden sollten, mit der Civil Religion, welche wiederum einen Komplex erinnerungskultureller Praktiken zur (Heiligen-)Verehrung der Nation umfasst, evident. Die Reihe an Formen, in denen sich die Civil Religion manifestiert, wie etwa den sacred texts, lässt sich besonders mit den Worten Hebels erfassen: Post-Civil War America saw the further pluralization and commercialization of the U.S.-American landscape of performative memory." ${ }^{10}$ Speziell der dabei angedeutete Aspekt der „performativen Erinnerung” steht in einem engen Zusammenhang mit Lincolns Forderung nach Taten („require us faithfully to perform ${ }^{\prime 81}$ ), und zwar in Form eines Treueschwurs oder -gelöbnisses. Eine Diskussion der Civil Religion, der "performativen Erinnerung“ und Lincolns Bestrebungen zur Förderung einer nationalen Einheit erfordert deshalb eine Berücksichtigung des sogenannten „Pledge of Allegiance". Denn innerhalb dieser "Landschaft der performativen Erinnerung" stellt dieser in seiner Bedeutung und Dimension einen Faktor dar, der seinesgleichen sucht.

\subsection{Der "Pledge of Allegiance" als Sonderbeispiel}

Der "Pledge of Allegiance" ist ein Treuegelöbnis in den USA, welches bei bestimmten öffentlichen oder politischen Ereignissen, Veranstaltungen und Zeremonien abgelegt wird. Eine erstmalige Veröffentlichung davon gab es im Jahr 1892 in einer Jugendzeitschrift. Nach kleineren Textänderungen wurde es im Jahr 1945 vom Kongress offiziell angenommen. ${ }^{82}$ Die aktuelle Version im United States Code lautet: II pledge allegiance to the Flag of the United States of America, and to the Republic for which it stands, one Nation under God, indivisible, with liberty and justice for all."83 Der "Pledge" hat in der mit Lincoln in Verbindung stehenden Erinnerungskultur und der Konstruktion von nationaler Einheit eine unvergleichbar große Bedeutung. Als performativer Akt der Erinnerung bildet er selbst einen „Erinnerungsort” im weitesten Sinne. Erinnerungsorte können grundsätzlich

„geographische Orte, Gebäude, Denkmäler und Kunstwerke ebenso umfassen wie historische Persönlichkeiten, Gedenktage, philosophische und wissenschaftliche Texte oder symbolische Handlungen"84

sein. Darüber hinaus seien „alle kulturellen Phänomene (ob material, sozial oder mental), die auf kollektiver Ebene bewusst oder unbewusst in Zusammenhang mit Vergan-

80 Hebel, Sites, S. 53.

81 Lincoln, Lyceum, S. 9.

82 Udo Sautter, Die Vereinigten Staaten. Daten, Fakten, Dokumente, Tübingen-Basel 2000, S. 205.

83 Legal Information Institute, U.S. Code, 26. 12. 2013, [https://www.law.cornell.edu/uscode/text/4/4], eingesehen 17. 10. 2018.

84 Erll, Gedächtnis, S. 25. 
genheit oder nationaler Identität gebracht werden" als Erinnerungsorte zu fassen. ${ }^{85}$ Gemäß dieser Definition stellt der "Pledge of Allegiance" eine soziale symbolische Handlung dar, die auf kollektiver Ebene bewusst getätigt wird. Allerdings ist sie wohl eher unbewusst mit der Vergangenheit, konkret mit Lincoln, verknüpft und konstruiert eine weitgehend unhinterfragte "nationale Identität" und Einheit. Wie Kao und Copulsky anmerken, wird der "Pledge" von den meisten US-Amerikaner*innen verehrt und täglich von Millionen von Schüler*innen und deren Lehrpersonen in den Schulen des ganzen Landes wiedergegeben. Zusätzlich beschließe er jede Einbürgerungszeremonie in den USA, eröffne jede Sitzung des Senats und werde bei verschiedensten Verbänden und Organisationen aufgesagt. ${ }^{86}$ Deshalb sei er das bekannteste Element US-amerikanischer ziviler Zeremonien und habe auch religiöse Bedeutung erlangt. ${ }^{87}$ Des Weiteren beschreiben sie ihn als

„very visible, indeed, perhaps the most ubiquitous, instance of American civil religion - a practice expressly endorsed by the state and ritually re-enacted nearly every day when public schools are in session".88

Der "Pledge" ist demnach aufgrund seiner täglichen Verwendung in Schulen das wohl allgegenwärtigste Beispiel in der Civil Religion. Diese Beobachtung ist besonders signifikant, da wie bereits angedeutet der "Pledge" in der einschlägigen Literatur über Civil Religion wenig bis gar nicht beachtet wird. Als beinahe täglich ausgeübtes Ritual hat er als performativer Akt jedoch eine Sonderstellung in der Glaubensausübung der Civil Religion. Im Sinne Rüsens synthetisiert er somit die drei Zeitdimensionen, gibt einen „übergreifenden Sinn“ und wird zum „(intentionale[n]) Bestandteil der menschlichen Lebenspraxis".89 Neben den Kategorien sacred places, sacred people oder sacred texts bildet er als sacred act das heilige Gebet der „zivilen Religion“. Es liegt in der Natur und Funktion eines Gebetes, eine Hingabe zu etwas auszudrücken, eine Beziehung zu pflegen und eine Haltung oder Anschauung einzunehmen und diese zu bekräftigen. Die tägliche Wiederholung macht die Handlung zu einem Ritual und verstärkt die Wirkung des Aktes, wie Schwartz konstituiert: „Without ritual, belief would be less comprehensible and moving. Ritual dramatizes belief and unifies people around it."190 Der Charakter des Rituals ermöglicht somit eine Inszenierung des Glaubens und vereint Menschen darin. Die performative Macht der Sprache spielt dabei eine besondere Rolle, wie Austins Sprechakttheorie betont:

„Die Funktion von Sprache erschöpft sich nicht darin, Sachverhalte abzubilden; sie wird auch dazu eingesetzt, um Wirklichkeiten und Sachverhalte zu schaffen, die ohne das Sprechen nicht eintreten könnten." ${ }^{\text {91 }}$

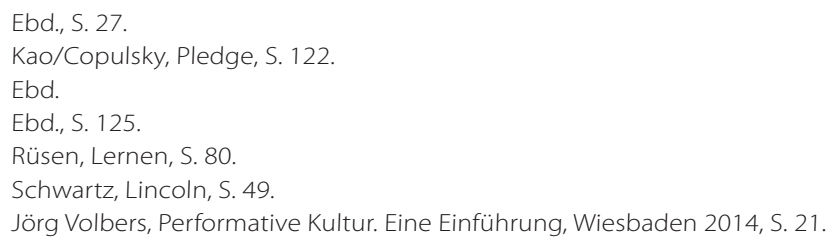


Die sprachliche Handlung konstruiert damit eine soziale Wirklichkeit auf der Mikroebene des Individuums. Speziell für das Ziel, ein Gefühl von nationaler Einheit herzustellen, ist der "Pledge" als "Glaubensbekenntnis" besonders relevant. Insofern steht er unmittelbar in einem Zusammenhang mit Lincoln und seinen Ideen zur Herstellung einer nationalen Einheit. Einerseits ist diese Verknüpfung in seinen Forderungen nach einer Performanz in einem Treueschwur (swear/pledge) innerhalb einer political religion in der zentralen Rede des Jahres 1838 zu sehen. Andererseits stellt der Wortlaut indivisible im Text des „Pledge of Allegiance“ eine direkte Referenz zur gesamten historischen Erfahrung des Bürgerkrieges dar. Die Bekräftigung der Unteilbarkeit der Nation vereint wie kein anderer Ausdruck die grundlegenden Differenzen, welche mit der Sezession und dem militärischen Konflikt einhergingen. Dadurch internalisiert sich die religiöse Überzeugung eines homogenen, einheitlichen Bildes der Nation. Als Sonderbeispiel der Civil Religion setzt der "Pledge“ Lincolns Forderung nach einem Treuegelöbnis in die Tat um, stellt ein Gefühl nationaler Einheit her und fungiert innerhalb der Civil Religion als das tägliche Gebet. Dieses bekräftigt eine Treue zur Nation und wortwörtlich zu den Werten, für die sie steht. Darüber hinaus gilt er unbewusst als Erinnerungsort, nämlich als symbolische Handlung, welche mit indivisible auf den Bürgerkrieg und die (Un-) Teilbarkeit der Nation Bezug nimmt.

Der US Code regelt die Durchführung des „Pledge of Allegiance“ mit der Vorschrift zur Ausrichtung zur Flagge mit der rechten Hand auf dem Herz und erinnert damit an einen militärischen Fahnenappell. ${ }^{92}$ Dieser Umstand und der Charakter des "Pledge“ erfordern deshalb eine kurze Kontextualisierung. Die Entstehung des Treueschwurs ist wiederum eng mit dem Bürgerkrieg bzw. mit der Zeit danach verknüpft. Durch die Spaltung der Nation und die Wiedervereinigung in der Reconstruction habe es in der Gesellschaft Angst vor der zunehmenden Einwanderung sowie die Befürchtung gegeben, dass sich Immigrant*innen nicht an die nationale Kultur anpassen würden. ${ }^{93}$ So seien in den 1880er-Jahren die Schulen für die Vermittlung von Patriotismus instrumentalisiert worden. ${ }^{94}$ Im Zuge eines Preisschreibens zur Vorbereitung der Feierlichkeiten der Columbus Day Gedenkfeier im Jahr 1892 schrieb Francis Bellamy einen Text, welcher später zum „Pledge of Allegiance“ werden sollte. Daneben existierte allerdings auch ein anderer Vorschlag für einen Treueschwur, nämlich jener von George T. Balch. ${ }^{95}$ Dieser sollte - wie der von Bellamy vorgeschlagene Akt - mit dem zur Flagge ausgestreckten rechten Arm, Hand flach nach unten, mit folgendem Wortlaut abgelegt werden: „We give our Heads! and our Hearts! to our Country! One Country! [One] Language! One Flag!" ${ }^{\prime 96}$ Damit weist seine Ausführung aus heutiger Perspektive in zweifacher Hinsicht ikonische Merkmale einer faschistischen Ästhetik auf. Einerseits ist die Handbewegung des römischen Grußes dieselbe, wie sie später Adolf Hitler und das NS-System zum öffentlich-performativen Identifikationsmerkmal machten. Ande-

92 Legal Information Institute, U.S. Code, 26. 12. 2013.

93 Leisa A. Martin, Examining the Pledge of Allegiance, in: The Social Studies 99 (2008), Nr. 3, S. 127-131, hier S. 127.

94 Ebd., S. 127-128.

95 Ebd., S. 128.

96 George T. Balch, Manual of the Patriotic Salute, in: The Journal of Education 38 (1893), Nr. 16, S. 271. 
rerseits spiegelt dieser Treueschwur sprachlich mit der Beschwörung eines monolingualen Einheitsstaates die bekannte Anapher der NS-Propagandasprache „Ein Volk! Ein Reich! Ein Führer!" wider. Zusätzlich nimmt sich dieser Schwur thematisch des LincolnMythos an, indem er mit "We give our Heads! and our Hearts!" die mit Lincoln, seinem Tod und dem Bürgerkrieg einhergehende Idee der Opferbereitschaft propagiert. ${ }^{97}$ In weiterer Folge setzte sich jedoch Bellamys Vorschlag durch, sodass inn am 21. Oktober 1892 bei der landesweiten National Columbus Public School Celebration über zwölf Millionen Menschen an Schulen aufsagten, woraufhin er sich als national tradition verfestigte. ${ }^{98}$ Während des Zweiten Weltkrieges seien aufgrund der Ähnlichkeit mit dem Hitlergruß Bedenken über die Gestik aufgekommen, weshalb manche Schulen auf die Handbewegung verzichteten. ${ }^{99}$

In Anbetracht seiner Tradition und Entstehungsgeschichte kann der „Pledge of Allegiance" als performativer Akt einer nationalistischen und faschistoiden Kultur betrachtet werden, welcher v. a. den öffentlichen Raum der Schule durchdringt. Interessanterweise beschreibt Bellah das öffentliche Schulsystem als „a particularly important context for the cultic celebration of the civil rituals".100 Die Rolle der Schule als wesentliche Sozialisationsinstanz darf dabei in Hinblick auf eine mögliche Indoktrination hin zu einem verstärkten Nationalismus oder (vorauseilenden) Gehorsam nicht unterschätzt werden. Vor allem im Sinne einer Trennung von Staat und Religion in einer globalisierten, pluralistischen Demokratie mit heterogenen Gesellschaften und Identitäten erscheint der Anspruch auf eine nationale Einheit in einer religiösen Beschwörung der Nation besonders problematisch. Dies erfordert eine Reflexion des Konstrukts der Nation, um schließlich einem demokratischen Bildungsanspruch, aufgeklärte, mündige und kritische Staatsbürger*innen zu erziehen, gerecht zu werden. Den Versuch einer kritischen Betrachtung der Topoi von nationaler Einheit, Faschismus und Indoktrination unternimmt die dystopische US-Serie The Man in the High Castle (2015-). Die von Amazon Studios produzierte, auf dem Roman von Philip K. Dick basierende Serie behandelt eine Alternativgeschichte, in welcher die Achsenmächte den Zweiten Weltkrieg gewannen und das NS-System in Teilen der USA etablierten. In einem Teaser zur zweiten Staffel wird der "Pledge of Allegiance" in diesen Kontext, nämlich in den Alltag eines Schulsettings, eingebettet. In der (Bild-)Sprache des Nationalsozialismus rezitiert eine US-Schulklasse in Ähnlichkeit zum realen, im US-Code festgeschriebenen Treuegelöbnis einen Schwur:

"I swear I will observe the law, conscientiously fulfil my duties at home and school, be faithful and obedient, and pledge absolute allegiance until death to the leader of the Nazi empire, Adolf Hitler."101

97 Siehe zur Idee des Opfers: Nagler, Lincoln, S. 192; Bellah, Religion, S. 10.

98 Martin, Examining, S. 128.

99 Ebd.

100 Bellah, Religion, S. 11.

101 Amazon Prime Video, The Man in the High Castle Season 2 - We Pledge Allegiance ... | Prime Video, Youtube, 4. 11. 2016, [https://www.youtube.com/watch?v=H4UHqFoB_5k], eingesehen 19. 10. 2018; Hervorhebung des Verfassers. 
Gefolgt von drei vom Hitlergruß begleiteten „Sieg heil!“-Rufen erzeugt diese kurze Sequenz in ihrer filmischen Sprache eine eindringliche Repräsentation des „Pledge of Allegiance". Auf besondere Weise zeigt sie die subtile Ideologie und mögliche Gefahren des Treueschwurs auf. Zudem drückt sie die Notwendigkeit aus, die Mechanismen einer (nationalistisch orientierten) Erinnerungskultur und Geschichtspolitik zu beleuchten und kritisch zu hinterfragen. Einen solch kritischen Zugang zu den Heiligtümern der Nation diskutiert ein abschließender Ausblick.

\section{Schluss und Ausblick: Kritik und die Heiligtümer der Nation}

Abschließend lässt sich zur Beantwortung der Forschungsfragen festhalten, dass Lincoln ein sehr ausgeprägtes Bewusstsein für die Rolle der Erinnerung als nation building hatte und dieses in seinen Reden zu fördern versuchte. In der politischen Kultur und Öffentlichkeit spiegelt sich dies im Konzept der Civil Religion wider. In den darin enthaltenen Heiligtümern der Nation nimmt der "Pledge of Allegiance" eine Sonderrolle ein, da er als Treuegelöbnis einen performativen Akt auf der Mikroebene darstellt, wesentliche Aspekte des US-amerikanischen kollektiven Gedächtnisses vereint und ein Gefühl von Einheit im Sinne Lincolns herstellt.

Vor allem der "Pledge of Allegiance“ verdeutlicht als in der Verfassung verankerte und täglich ausgeübte „Institution" der Erinnerungskultur die Herausforderungen einer "nationalen Einheit" in der Gegenwart. Diese manifestieren sich in einer Kritik an den Heiligtümern der Nation in öffentlichen Handlungen, in denen Individuen von der Norm abweichen und die Struktur von Ritualen durchbrechen. Eine Form der Kritik äußerte sich etwa in Fällen von religiösen Gruppierungen wie den Zeugen Jehovas, die sich aus Gewissensgründen weigerten, den „Pledge of Allegiance” abzulegen. Als Folge sei es zu rechtlichen und teilweise gewaltsamen Auseinandersetzungen gekommen. ${ }^{102} \mathrm{KaO}$ und Copulsky halten zur Problematik der Civil Religion passenderweise fest: „Insofar as such rituals strive to promote unity, they do so at the risk of estranging some citizens from full participation in the polity."103 Dabei geht es im Kern um genau diese Frage nach der Partizipation im Gemeinwesen. Diese Partizipation kann auch darin zum Ausdruck kommen, wie die national hegemonialen Geschichtsnarrative unterschiedliche Identitäten in- bzw. exkludieren. Dies äußerte sich in der Kritik am Heiligtum der Nationalhymne, wie das Beispiel von Harper Nielsen in Australien zeigt. Die neunjährige Schülerin weigerte sich, für die Nationalhymne aufzustehen, da jene mit ihrem Text die indigene Bevölkerung Australiens ignoriere. Nach ihrem Protest war Nielsen mit heftigen Beleidigungen konfrontiert. ${ }^{104}$ Ein weiteres Beispiel ist der NFL-Spieler Colin Kaepernick. Dieser kniete sich während des kollektiven Zelebrierens der Nationalhymne bei American-Football-Spielen hin und drückte damit seinen Protest gegen Rassismus und Polizeigewalt aus. Diese Aktion entwickelte sich zu einer weitreichenden Protestbewe-

102 Kao/Copulsky, Pledge, S. 141.

103 Ebd.

104 Kevin Rawlinson, Rightwing Australian politicians attack girl, 9, over anthem protest, in: The Guardian, 13. 9. 2018, [https://www.theguardian.com/education/2018/sep/12/rightwing-australian-politicians-criticise-girl-9-in-rowover-anthem], eingesehen 29. 10. 2018. 
gung, führte zu nationalen Debatten über "(un-)patriotisches Verhalten" und brachte Kaepernick öffentliche Beschimpfungen und Diskreditierung ein. ${ }^{105}$ Unter anderem äußerte US-Präsident Donald Trump mehrfach seinen Unmut über das für ihn entwürdigende und respektlose Verhalten gegenüber den „unantastbaren“ Heiligtümern der Nation. Beispielsweise betonte er in seiner Rede zur Lage der Nation 2018, die US-Flagge zu ehren, den „Pledge of Allegiance“ mit der Hand auf dem Herz durchzuführen und bei der Nationalhymne stolz erhoben zu stehen. ${ }^{106}$

Diese Beispiele zeigen die Herausforderungen einer Civil Religion und einer Beschwörung von nationaler Einheit auf. Im Kontext des 19. Jahrhunderts hatten Lincolns Bemühungen, eine nationale Einheit herzustellen, in einer patriarchalischen und hegemonialen weißen Gesellschaft wohl noch andere Implikationen und Konsequenzen. Doch in einer von emanzipatorischen Bestrebungen geprägten Nation, welche Demokratie im 21. Jahrhundert als Gesellschafts- und Lebensform versteht und gleichzeitig politisch oder sozial motivierte Kritik kategorisch verurteilt, führt die Verehrung von Phrasen wie liberty and justice for all zu erheblichen Widersprüchen. Die Freiheit beinhaltet scheinbar nicht das Recht, Kritik an der Nation sowie ihren Heiligtümern zu üben und sich für mehr "Gerechtigkeit für alle" einzusetzen. Eine moderne, pluralistische Demokratie braucht somit nicht nur das Recht zu einer gewissen Form des Ungehorsams als Ausdruck des Protests, sondern auch eine (selbst-)kritische Debatte über Partizipation, Identität und Geschichtskultur. Im Sinne der Public History geht es um die "Aushandlung von Geschichte in der Öffentlichkeit" als eine "macht- und bedeutungsvolle Angelegenheit“.107 Dabei kann eine „inklusive Erinnerungs- und Geschichtskultur" dazu beitragen, den exkludierenden, "hegemonialen Gedenkrahmen“108 der Nation für moderne Migrationsgesellschaften zu öffnen und eine Demokratisierung der Gesellschaft und ihrer Geschichte(n) zuzulassen. Gerade für die USA, die als die älteste Demokratie der Welt gelten, wäre das Zulassen einer solchen Vielfalt in der nationalen Erinnerungskultur ein gemeinsames Projekt, dem Motto e pluribus unum einen Schritt näherzukommen und den eigenen Ansprüchen gerechter zu werden.

\section{Quellen- und Literaturverzeichnis}

Amazon Prime Video, The Man in the High Castle Season 2 - We Pledge Allegiance ... I PrimeVideo, Youtube, 4.11.2016, [https://www.youtube.com/watch?v=H4UHqFoB_5k], eingesehen 19. 10. 2018.

105 Clark Mindock, Taking a knee: Why are NFL players protesting and when did they start to kneel?, in: The Independent, 4. 9. 2018, [https://www.independent.co.uk/news/world/americas/us-politics/taking-a-knee-national-anthemnfl-trump-why-meaning-origins-racism-us-colin-kaepernick-a8521741.html], eingesehen 29. 10. 2018.

106 CNBC, President Donald Trump Delivers the 2018 State of the Union Address (Full) | CNBC, Youtube, 30. 1. 2018, [https://www.youtube.com/watch?v=DfA03HQbj7E], eingesehen 29. 10. 2018, Min. 19:57-21:59, v. a. Min. 21:11-21:30; The White House, President Donald J. Trump's State of the Union Address, 30. 01. 2018, [https:// www.whitehouse.gov/briefings-statements/president-donald-j-trumps-state-union-address/], eingesehen 29. 10. 2018.

107 Lücke/Zündorf, Einführung, S. 52.

108 Ebd, S. 53. 
Anderson, Benedict, Imagined Communities. Reflections on the Origin and Spread of Nationalism, London-New York 2006.

Balch, George T., Manual of the Patriotic Salute, in: The Journal of Education 38 (1893), Nr. 16, S. 271.

Barrett, Jenny, Shooting the Civil War. Cinema, History and American National Identity, London 2009.

Bellah, Robert, Civil Religion in America, in: Daedalus 96 (1967), Nr. 1, S. 1-21.

Berg, Manfred, Geschichte der USA, München 2013.

Blight, David W., Race and Reunion. The Civil War in American Memory, Cambridge 2001.

Boime, Albert, Patriarchy Fixed in Stone. Gutzon Borglum's "Mount Rushmore", in: American Art 5 (1991), Nr. 1, S. 142-167.

CNBC, President Donald Trump Delivers the 2018 State of the Union Address (Full) | CNBC, Youtube, 30. 1. 2018, [https://www.youtube.com/watch?V=DfA03HQbj7E], eingesehen 29. 10. 2018.

Dippel, Horst, Geschichte der USA, München 1996.

Donald, David Herbert, Lincoln, New York 1995.

Edifice, in: English Oxford Dictionary Online, o. D., [https://en.oxforddictionaries. com/ definition/edifice], eingesehen 28. 9. 2018.

Emmerich, Alexander, Geschichte der USA, Stuttgart 2008.

Erll, Astrid, Kollektives Gedächtnis und Erinnerungskulturen. Eine Einführung, StuttgartWeimar $2011^{2}$.

Hebel, Udo J., Sites of Memory in U.S.-American Histories and Cultures, in: Astrid Erll/ Ansgar Nünning (Hrsg.), Cultural Memory Studies. An International and Interdisciplinary Handbook, Berlin-New York 2008, S. 47-60.

Hvithamar, Annika/Warburg, Margit, Introducing Civil Religion, Nationalism and Globalisation, in: Annika Hvithamar/Margit Warburg/Brian Arly Jacobsen (Hrsg.), Holy Nations and Global Identities. Civil Religion, Nationalism, and Globalisation, Leiden-Boston 2009, S. 1-17.

Kao, Grace Y./Copulsky, Jerome E., The Pledge of Allegiance and the Meanings and Limits of Civil Religion, in: Journal of the American Academy of Religion 75 (2007), Nr. 1, S. $121-149$.

Legal Information Institute, U.S. Code, 26. 12. 2013, [https://www.law.cornell.edu/ uscode/text/4/4], eingesehen 17. 10. 2018.

Lincoln, Abraham, Address to the Young Men's Lyceum of Springfield, Illinois, January 27, 1838, in: David S. Reynolds (Hrsg.), Lincoln's Selected Writings, New York-London 2015, S. 8-14. 
Ders., First Inaugural Address, March 4, 1861, in: Reynolds (Hrsg.), Selected Writings, S. 228-234.

Ders., House Divided Speech at Springfield, Illinois, 1858, in: Reynolds (Hrsg.), Selected Writings, S. 129-135.

Lücke, Martin/Zündorf, Irmgard, Einführung in die Public History, Göttingen 2018.

Martin, Leisa A., Examining the Pledge of Allegiance, in: The Social Studies 99 (2008), Nr. 3, S. 127-131.

Mindock, Clark, Taking a knee: Why are NFL players protesting and when did they start to kneel?, in: The Independent, 4. 9. 2018, [https://www.independent.co.uk/news/ world/ americas/us-politics/taking-a-knee-national-anthem-nfl-trump-why-meaningorigins-racism-us-colin-kaepernick-a8521741.html], eingesehen 29. 10. 2018.

Müller, Kurt, Abraham Lincoln. The Emergence, Appropriation, and Contestation of an American Icon, in: Günther Leypoldt/Bernd Engler (Hrsg.), American Cultural Icons. The Production of Representative Lives, Würzburg 2010, S. 383-409.

Nagler, Jörg, Abraham Lincoln (1861-1865). Bewahrung der Republik und Wiedergeburt der amerikanischen Nation, in: Jürgen Heideking (Hrsg.), Die amerikanischen Präsidenten. 42 historische Portraits von George Washington bis George W. Bush, München $2002^{3}$, S. 176-193.

National Park Service, 6. 7. 2011, [https://www.nps.gov/moru/learn/news/thousandscelebrate-at-the-shrine-of-democracy.htm], eingesehen 17. 10. 2018.

Ders., 19. 4. 2017, [https://www.nps.gov/moru/learn/ historyculture/index.htm], eingesehen 17. 10. 2018.

Pandel, Hans-Jürgen, Geschichtsunterricht nach PISA. Kompetenzen, Bildungsstandards und Kerncurricula, Schwalbach/Ts. 2005.

Parsons, Gerald, Perspectives on Civil Religion, Burlington-Sydney 2002.

Rawlinson, Kevin, Rightwing Australian politicians attack girl, 9, over anthem protest, in: The Guardian, 13. 9. 2018, [https://www.theguardian.com/education/2018/sep/12/rightwing-australian-politicians-criticise-girl-9-in-row-over-anthem], eingesehen 29. 10. 2018.

Reeh, Niels, American Civil Religion as State-Mythology, in: Annika Hvithamar/Margit Warburg/Brian Arly Jacobsen (Hrsg.), Holy Nations and Global Identities. Civil Religion, Nationalism, and Globalisation, Leiden-Boston 2009, S. 79-97.

Rüsen, Jörn, Historisches Lernen. Grundlagen und Paradigmen, Köln-Weimar-Wien 1994. Sautter, Udo, Die Vereinigten Staaten. Daten, Fakten, Dokumente, Tübingen-Basel 2000. Schaser, Angelika, Nation, Identität und Geschlecht. Nationalgeschichtsschreibung und historische Frauen- und Geschlechterforschung, in: Karin Hagemann/Jean Quataert (Hrsg.), Geschichte und Geschlechter. Revisionen der neueren deutschen Geschichte, Frankfurt am Main 2008, S. 64-91. 
Schwartz, Barry, Abraham Lincoln and the Forge of National Memory, Chicago-London 2000 .

Stöver, Bernd, Geschichte der USA. Von der ersten Kolonie bis zur Gegenwart, München $2017^{2}$.

The White House, President Donald J. Trump's State of the Union Address, 30.1.2018, [https://www.whitehouse.gov/briefings-statements/president-donald-j-trumps-stateunion-address/], eingesehen 29. 10. 2018.

Tocqueville, Alexis de, Democracy in America and Two Essays on America, London 2003.

Volbers, Jörg, Performative Kultur. Eine Einführung, Wiesbaden 2014.

Benedikt Kapferer ist Lehramtsstudent der Fächer Geschichte, Sozialkunde und Politische Bildung und Englisch an der Universität Innsbruck. benedikt.kapferer@ student.uibk.ac.at

\section{Zitation dieses Beitrages}

Benedikt Kapferer, Die Heiligtümer der Nation. Abraham Lincoln, Erinnerungskultur und die "nationale Einheit" der USA, in: historia.scribere 11 (2019), S. 135-158, [http:// historia.scribere.at], eingesehen 17.6.2019 (=aktuelles Datum).

(C) Creative Commons Licences 3.0 Österreich unter Wahrung der Urheberrechte der Autorlnnen. 